\title{
H7N6 Low Pathogenic Avian Influenza outbreak in commercial turkey farms in Chile caused by a native South American Lineage
}

Mathieu C. ${ }^{1 *}$, Gonzalez A. ${ }^{1}$, Garcia A. ${ }^{1}$, Johow M. ${ }^{1}$, Badia C. ${ }^{1}$, Jara C. ${ }^{1}$, Nuñez P. ${ }^{1}$, Neira V. ${ }^{2}$, Montiel N.A. ${ }^{3}$, Killian M.L. ${ }^{3}$, Brito B.P. ${ }^{2,4 *}$

${ }^{1}$ Servicio Agrícola y Ganadero (SAG), Laboratorio y Estación Cuarentenaria de Lo Aguirre, Santiago, Chile.

${ }^{2}$ Departamento de Medicina Preventiva, Facultad de Ciencias Veterinarias y Pecuarias, Universidad de Chile, La Pintana, Santiago, Chile.

${ }^{3}$ National Veterinary Services Laboratories, Science, Technology and Analysis Services, Veterinary Services, Animal and Plant Health Inspection Service, U.S. Department of Agriculture, Ames, lowa, USA

${ }^{4}$ The ithree institute, University of Technology Sydney, PO Box 123, Broadway, NSW 2077, Australia

Running title: H6N7 LPAI influenza in Chile

Keywords: Influenza in birds, Disease Outbreaks, Viruses, Phylogeny, Poultry

*Correspondence: christian.mathieu@sag.gob.cl,

barbara.britorodriguez@uts.edu.au

\section{SUMMARY}

In December of 2016, low pathogenic avian influenza (LPAI) caused by an H7N6 subtype was confirmed in a grow-out turkey farm located in Valparaiso Region, Chile. Depopulation of exposed animals, zoning, animal movement control and active surveillance were implemented to contain the outbreak. Two weeks later, 
a second turkey grow-out farm located $70 \mathrm{~km}$ north of the first site was also infected by H7N6 LPAI, which subsequently spilled over to one backyard poultry flock. The virus involved in the outbreak shared a close genetic relationship with viruses collected from Chilean aquatic birds' viruses. The A/turkey/Chile/2017(H7N6) LPAI virus belonged to a native South American lineage. Based on the $\mathrm{H} 7$ and most of the internal genes phylogenies, these viruses were also closely related to the viruses that caused a highly pathogenic avian influenza outbreak in Chile in 2002. Results from this study help understand the regional dynamics of influenza outbreaks, highlighting the importance of local native viruses circulating in the natural reservoir hosts.

\section{INTRODUCTION}

Outbreaks caused by avian influenza viruses (AIV) have major impacts in the poultry industry. Each year, events of highly pathogenic avian influenza (HPAI), and low pathogenic avian influenza (LPAI) are reported by different countries regardless of their overall animal health status and strategies to prevent the introduction of foreign animal diseases. These outbreaks cause high economic losses to the poultry industry and governments, due to elimination of all exposed animals, deployment of resources for surveillance and disease control strategies, as well as international trade restrictions (Djunaidi \& Djunaidi, 2007; Otte, Hinrichs, Rushton, D, \& D, 2008). Disease prevention is complex because many wild aquatic birds are a natural reservoir of influenza A viruses (Vandegrift, Sokolow, Daszak, \& Kilpatrick, 2010).

The H5 and H7 subtypes are known for their potential to mutate to HPAI, typically after infecting poultry, resulting in severe disease and high mortality (Alexander, 2000). Among the outbreaks caused by $\mathrm{H} 7$, special attention has 
been given to two HPAI events. The first one was caused by an H7N9 virus that emerged in China in 2013. This virus is highly poultry adapted and spread widely in China, mutating to a highly pathogenic form during late 2016. The H7N9 virus that caused this outbreak is particularly concerning because, besides causing severe disease in poultry, it has also been responsible for more than 1,600 cases of illness in humans (CDC, 2018; Jiao et al., 2018; Ke et al., 2017). The second event also, caused by an H7N9 subtype was reported in March 2017 in the United States. This outbreak was caused by a North American wild bird lineage virus, which was genetically unrelated and epidemiologically different to the Asian H7N9 virus. Multiple LPAI introductions of this virus from wild birds into poultry were inferred based in epidemiological and molecular investigations. Mutation into HPAI occurred one time and resulted in the culling of more than 200,000 animals (Lee, Torchetti, Killian, Berhane, \& Swayne, 2017). This event was contained and concluded six months after initial detection (OIE, 2018a).

The genetic diversity of AIV in South America has not been described in detail compared to that in North America. Regional and local dynamics of AIV have been associated to local South American and North American lineages and their transmission through migration routes that connect North and South America, especially the Pacific, Central and Mississippi Flyways. However, the information of subtypes and lineages circulating is not uniform across the continent, and throughout different time periods. Of the previously reported AIVs in Latin America, $43.7 \%$ belong to migratory birds, $28.1 \%$ to local wild birds, and $28.1 \%$ to poultry (Afanador-Villamizar, Gomez-Romero, Diaz, \& Ruiz-Saenz, 2017). Additionally, several influenza subtypes have been detected in Chilean wild birds, including $\mathrm{H} 5$ and $\mathrm{H} 7$ subtypes, highlighting the risk for poultry production (Bravo-Vasquez et al., 2016; Jimenez-Bluhm et al., 2018; Mathieu et al., 2015). 
Chilean poultry has been affected by HPAI only once, in 2002. The outbreak was caused by an HPAI A/chicken/Chile/2002(H7N3) virus that resulted in economic losses of over 31 million USD and 635,000 animals dead or culled (AfanadorVillamizar et al., 2017; Max, Herrera, Moreira, \& Rojas, 2007; Spackman, McCracken, Winker, \& Swayne, 2006). Other AIV impacting poultry in Chile include an outbreak in turkeys in Valparaiso region in 2009, which was caused by the H1N1 pandemic ( $\mathrm{pH} 1 \mathrm{~N} 1$ ) human virus. This event was evidenced by decreased egg production and shell quality, and was reported only two months after the first pH1N1 detection in humans in Chile (Mathieu et al., 2010). In 2011, LPAI H4N8 was found by serologic surveillance in a grow-out turkey farm, also in the region of Valparaiso. The presence of this AIV was confirmed by isolation in embryonated eggs. There was no evident clinical disease at the farm and rapid detection prevented further spread of the virus (SAG, 2011).

In this study, we describe the epidemiological investigations of an LPAI H7N6 outbreak in Chilean poultry in late 2016 and early 2017. We characterized the viruses using whole genome sequencing and reconstructed their phylogeny using Bayesian time divergence estimation, to infer their genetic relation with North and South American AIVs. These results highlight the importance of characterization and molecular surveillance of local South American AIV strains to understand the viruses that pose a major risk to domestic poultry.

\section{METHODS}




\section{Description of the outbreak}

On December $26^{\text {th }}$ of 2016 a grow-out turkey house (Farm A) in the region of Valparaiso reported clinical respiratory disease. On the following day, respiratory signs increased in severity. Noticeable, this farm had undergone serological (ELISA) AIV testing $(n=60)$ on December $20^{\text {th }}$, yielding negative results. On December $29^{\text {th }}$ and following the diagnosis of clinical disease, the poultry company notified positive AIV ELISA test results to the Chilean Agricultural and Livestock Service (Servicio Agricola Ganadero; SAG).

On December $30^{\text {th }}$, SAG officially confirmed the presence of AIV by real time RTPCR (AI matrix gene). Subsequently, H5 and H7 subtype real time RT-PCR assays were performed, confirming an H7 subtype. The samples were initially sent to a private laboratory (Macrogen, Inc) for partial sequencing of the HA cleavage site. The amino acid sequence was consistent with a LPAI virus (corresponding to the translated amino acid site: NVPEKPRTR/GLF). Characterization of the neuraminidase gene subtype was carried out by neuraminidase-inhibition assay at SAG, revealing a N6 subtype. Further characterization of the virus included sequencing and in vivo testing to confirm the pathotype, performed at NVSL and SAG.

Once the LPAI H7N6 outbreak was officially confirmed, immediate control measures were established. A control area consisting of two zones surrounding the infected premises were defined: (1) an infected zone within a $3 \mathrm{~km}$ radius of the infected premises where animal movement was restricted, and (2) a buffer zone within $7 \mathrm{~km}$ radius of the infected premises, where increased biosecurity and surveillance for avian influenza were carried out (USDA, 2015). Additionally, 
all breeders and grow-out farms belonging to the affected company throughout the country were tested for influenza (including the ones outside the buffer zone). Surveillance consisted in blood sample collection and testing by agar gel immunodiffusion (AGID) assay performed at the SAG official diagnostic laboratory. A total of 327,000 exposed animals in Farm A were culled.

By January $2^{\text {nd }}$, clinical respiratory disease was present in flocks in five out of 8 houses within Farm A. The animals presented a variety of gross lesions including bursitis, catarrhal to mucopurulent tracheitis, caseous airsacculitis, polyserositis, pericarditis/hydropericardium, congestion and pulmonary oedema, mucopurulent to caseous pneumonia, localized subcutaneous emphysema, mild splenomegaly, and pancreatitis. Perihepatitis was only found in one house in the Farm A.

At the national level, recent records with productivity data from all commercial farms were reviewed, focusing in mortality, meat production, and egg production curves. The objective was to determine any abnormalities and potential undetected cases using syndromic surveillance. The parameters from all commercial farms yielded within-expected production values.

On $17^{\text {th }}$ January of $2017,70 \mathrm{~km}$ north from the first affected farm (Valparaiso), a male turkey grow-out farm (Farm B) belonging to the same company, was visited by a veterinarian due to increased respiratory disease signs. SAG confirmed the presence of AIV and culled 35,572 exposed animals in the farm, which was subsequently cleaned and disinfected. Infected and buffer zones were put in place around the infected premises. Disposal of all carcasses was done by burial. In Farm B clinical gross lesions observed consisted of caseous sinusitis, 
catarrhal tracheitis, petechiae and ecchymosis focused on epicardium and coronary fat, fibrin-purulent pericarditis, purulent airsacculitis, pulmonary congestion/mucopurulent to caseous pneumonia, pleuritis, mild splenomegaly.

Epidemiological investigations concluded that the initial entry route of the virus into Farm A was through a breach in biosecurity which resulted in transmission from wild birds through faeces or contaminated water into one of the grow-out sites. Lateral transmission between Farm A and Farm B likely occurred due to an unreported breach in biosecurity by personnel, supplies or vehicles that were shared between Farm A and Farm B.

In January $28^{\text {th }}$ a backyard poultry farm within a household located in the defined buffer zone of Farm B tested positive to AIV by AGID but was negative to PCR testing. This farm had direct links through personnel with Farm B. All poultry in the household were culled.

Thereafter, all testing conducted in commercial farm inside and outside the control zones were negative to AIV. On June $9^{\text {th }}$, three months after finalizing stamping out of exposed and infected animals, Chile regained the OIE free status (OIE, 2017).

\section{Viral sequencing and phylogenetic analysis}

Complete genome sequencing attempts were made from oral and tracheal swab pools at the United States Department of Agriculture, Animal and Plant Health Inspection Service, National Veterinary Services Laboratories, Ames, Iowa. 
Briefly, viral RNA was extracted from samples using the MagMAX Viral RNA Isolation Kit (Ambion/ThermoFisher Scientific). Complementary DNA was synthesized by reverse transcription reaction using SuperScript III (Invitrogen/ThermoFisher Scientific). All eight segments were amplified by PCR and complete genome sequencing was conducted using the Illumina Miseq system. The Nextera XT DNA Sample Preparation Kit (Illumina) was used to generate multiplexed paired-end sequencing libraries, according to the manufacturer's instructions. The dsDNA was fragmented and tagged with adapters by Nextera XT transposase and 12-cycle PCR amplification. Fragments were purified on Agencourt AMpure XP beads (Beckman Coulter). The barcoded multiplexed library sequencing was performed using the 500 cycle MiSeq Reagent Kit v2 (Illumina). De Novo and directed assembly of genome sequences were carried out using the SeqMan NGen v.4 program. Viral sequences were submitted to GenBank (accession numbers MK424141-MK424219).

Phylogenetic analyses were performed separately for each segment. The closest references viruses were obtained by a BLAST (https://blast.ncbi.nlm.nih.gov/Blast.cgi) search and the 100 closest hits were included to reconstruct the phylogenetic tree. Sequences obtained for each segment and the corresponding reference sequences were aligned MUSCLE (Edgar, 2004). Redundant reference sequence discarded (identical or near identical sequences with similar collection dates and locations). Bayesian time divergence estimation using the $\mathrm{HKY}+\mathrm{G}[4]$ nucleotide substitution model and an uncorrelated relaxed clock was performed for all segments. Coalescent Bayesian Skyline tree prior was used to reconstruct the phylogeny of $\mathrm{H7}$ (segment 4) to allow for population size changes over time. For N6 (segment 6) exponential 
growth and Bayesian skyline coalescent tree priors were compared using path sampling and stepping-stone sampling marginal likelihood estimation (Baele, Li, Drummond, Suchard, \& Lemey, 2013). For the remaining segments $1,2,3,5,7$ and 8 (coding for PB2, PB1, PA, NP, M and NS respectively) a coalescent exponential growth tree prior was initially run. If the $95 \%$ high posterior density (95\% HPD) of the growth parameter included 0 (thus, meaning that the population size is not growing in time) a coalescent constant population tree prior was run and used for the final phylogeny, whereas if the parameter did not include 0 , the original run with the exponential growth tree prior used was used. The analyses were run in BEAST 1.8.4 (Drummond, Suchard, Xie, \& Rambaut, 2012). A total of 500000000 iterations were run, sampling every 50000 trees using Cipres computational resources (Miller, Pfeiffer, \& Schwartz, 2010). Traces of the parameters were checked for convergence and for effective sample size (ESS) $>200$. The maximum clade credibility (MCC) tree was annotated, burning the first $10 \%$ sampled trees, using TreeAnnotator and visualized using Figtree (http://tree.bio.ed.ac.uk/software/figtree/). Amino acid sequences were compared to the closest references to identify relevant mutations using MEGA7 (Kumar, Stecher, \& Tamura, 2016).

\section{RESULTS}

Phylogenies were estimated using coalescent constant population tree priors for PB2, PA, NP, M and NS coding segments. Bayesian skyline tree prior was used for reconstructing HA and NA phylogenies, while exponential growth was used to estimate the phylogeny of PB1 (Table 1). The estimated nucleotide substitution rate per site per year for each coding segment ranged between $1.96 \times 10^{-3}$ 
(95\%HPD $\left.1.52 \times 10^{-3}-2.44 \times 10^{-3}\right)$ and $3.98 \times 10^{-3}\left(95 \% H P D 2.83 \times 10^{-3}-5.26 \times 10^{-3}\right)$, which were the lowest and highest rates estimated for segment 7 (M) and 4 $(\mathrm{HA})$, respectively.

\section{HA coding segment}

All viruses from the 2017 Chilean turkey LPAI outbreak viruses were included in a monophyletic clade, which shared the closest ancestor with 2 viruses (A/yellow_billed_pintail/Chile/10/2014(H7N3), and A/yellow_billed_teal/Chile/9/2013(H7N6)) collected from wild local water birds. The tMRCA shared by the closest Chilean wild bird virus and the A/turkey/Chile/2002(H7N6) was estimated at 2012 (95\% HPD 2011-2013)

(Figure 1). The A/turkey/Chile/2017(H7N6) LPAI viruses were also related to AIV found in wild aquatic birds in Chile and Bolivia, and with the 2002 HPAI Chilean outbreak with a tMRCA estimated at 1995 (95\% HPD 1989-1999). Based on the HA phylogeny, these South American viruses were grouped in a unique cluster. The remaining closest reference, belonging to a North American avian lineage, that recently caused an HPAI outbreak in the United States, shared an earlier common ancestor estimated in 1944 (95\% HPD 1913-1969). Specific amino acid changes fixed in the LPAI A/turkey/Chile/2017(H7N6) compared to the closest references were in HA amino acid positions V42I, V96I, I191V, P524S, and a fixed mutation in Farm B sequences R316K (Relative to HPAI Chicken/Chile2002 which has a ten- amino acid insertion in positions 338-347; Table 2). Three of these specific amino acid changes are located in antigenic sites C, D and E (Supplementary file 1), which are not related to additional glycosylation sites. 


\section{NA coding segment}

Similar to HA coding segments, NA sequences from the A/turkey/Chile/2017(H7N6) LPAI outbreak are genetically related to a distinct group that includes viruses collected from Chilean wild birds in 2013 (H7 and H3 subtypes being the closest ones) with a tMRCA estimated at 2004 (95\% HPD 2000-2008) (Figure 2). This group is more genetically distant to viruses from North American avian compared to the HA and most internal genes' phylogenies, having a tMRCA estimated at 1869 (95\% HPD 1823-1911). Sequences from A/turkey/Chile/2017(H7N6) LPAI had three amino acid substitutions fixed compared to the closest references (amino acid positions T42P, V333A and N359S). We included only one sequence per sample to depict the phylogeny, however, a mixed population of NA sequences with and without stalk deletions (ranging between 23-27 amino acid long) were found in (pooled) samples collected from Farm A. All four samples from Farm B had sequences with the same 26 amino acid nucleotide stalk deletion between amino acid position 3257. This deletion was not associated with additional glycosylation sites in HA.

\section{Phylogeny of internal segments PB2, PB1, PA, NP, M and NS}

For the reconstructed phylogeny of internal segments, the closest reference sequences to the A/turkey/Chile/2017(H7N6) LPAI viruses were viruses collected from Chilean aquatic birds (Figure 3). The closest relationships for each segment of A/turkey/Chile/2017(H7N6) LPAI were with different viruses from a variety of HA and NA types (Table 1). PB1, PA, HA and NP internal genes coding segments of A/turkey/Chile/2017(H7N6) LPAI shared a similar tMRCA with 
A/chicken/Chile/2002(H7N3) HPAI estimated between 1992-1995. In terms of regional lineages, the $A /$ turkey/Chile/2017(H7N6) LPAI segments PB2, NP and M were grouped with avian viruses sampled mostly from South America and few from North America, whereas segments PA, HA, and NS clustered with sequences that were exclusively South American. The PA, NP and NS lineages to which A/turkey/Chile/2017(H7N6) LPAI belong, were related to equine viruses from as a result from a potential reassortment event that occurred sometime between 1942-1955 (Table 1). Interestingly, one of the M segments from the A/chicken/Chile/2002(H7N3) outbreak was distinct from other viruses collected during that epidemic (Figure 3).

Nucleotide mutations fixed in the A/turkey/Chile/2017(H7N6) LPAI viruses compared to close reference from local aquatic birds were found in PB1 (V344I, V444I) and T683I, in M2 (K18R, V27I) and in NP (V193I, T196I, M221V) as well as a Farm B specific fixed mutation in PB1 (R101G, N573H) and NP (M123I).

None of the segments were related to the pH1N1 viruses that caused an outbreak in turkeys during 2010, and which HA and NA were related to the 2009 pandemic virus.

\section{DISCUSSION}

In Chile, an outbreak of H7N6 LPAI (A/turkey/Chile/2017(H7N6)) was detected shortly after initial signs of respiratory disease were reported in a commercial turkey grow-out farm in December of 2016. Epidemiological investigations and phylogenetic analyses of viral sequence data were used to understand the source of the outbreak and characterize the lineage and the genetic relationships 
with reference viruses. All segments from the A/turkey/Chile/2017(H7N6) LPAI virus were related to viruses collected in previous years from wild birds in Chile. The A/turkey/Chile/2017(H7N6) LPAI virus grouped within the same lineage as the Chicken/Chile/2002(H7N3) HPAI, based on the phylogeny of HA and most genetic segments coding for internal proteins. The A/turkey/Chile/2017(H7N6) LPAI virus belongs to a native South American lineage, and are not closely related to other recent viruses reported from poultry in the Americas such as the subtype H7N9 that had caused outbreaks during 2017 in commercial chicken farms in Tennessee and Alabama in the United States (Lee et al., 2017), and the H7N3 that has caused sporadic HPAI outbreaks in Mexico since 2015 (OIE, 2018b). This South American lineage is also unrelated to the poultry adapted Asian H7N9 virus (CDC, 2018).

Control of AIV in poultry is complex and challenging. High biosecurity measures must be accomplished to prevent viruses that continuously circulate in wild birds from entering poultry operations. A wide diversity of influenza A viruses exist among water birds, which may persist in geographical areas where commercial and backyard poultry farms are located. A large number of wetlands throughout the extensive Chilean coast, provides an ecosystem where migratory and local wild water birds (including the main avian influenza reservoirs) co-exist (Jimenez-Bluhm et al., 2018). Small backyard production systems are also constantly exposed to transmission from wild birds and are, likely, sporadically infected (Bravo-Vasquez et al., 2016), representing another potential source of AIV for commercial farms. Additionally, transmission from human or other species also pose a risk for influenza outbreaks in poultry, such as the pH1N1 introduced from human into a commercial turkey farm in Chile in 2009 (Mathieu et al., 2010). 
A close ancestral relationship between viruses that caused the A/chicken/Chile/2002(H7N3) HPAI and the A/turkey/Chile/2017(H7N6) LPAI revealed the importance of this specific South American lineage virus as a risk for commercial poultry farms. Aquatic bird reservoirs have maintained this virus throughout the years, posing a risk to poultry farms that was not evidenced until the recent 2017 outbreak. Considering this H7N6 event, as well as the previous H7N3 in Chile, this local viral lineage has been the main source of AIV poultry outbreaks. However, the risk of introduction of other lineages into Chile should not be underestimated as there is a wide variety of local and North American lineages found in wild water birds.

Previous studies have supported that A/chicken/Chile/2002(H7N3), as well as other American AIV H7 subtype strains, need a lower infectious dose to cause clinical disease in turkey, compared to that needed to affect chicken (Spackman et al., 2010; Spackman et al., 2006). It is relevant to design targeted surveillance, as turkey may act as sentinels for emerging and more pathogenic AIV strains. In this particular event, clinical disease caused by LPAI in two turkey farms was rapidly detected and preventive measures were timely put in place to contain viral spread. Some viruses in Farm A and all viruses from Farm B had already acquired amino acid stalk deletion in its NA segment. These genetic changes have been associated to an adaptation to the domestic host and increased virulence and transmission (Campitelli et al., 2004; Li, Zu Dohna, Cardona, Miller, \& Carpenter, 2011; Sorrell, Song, Pena, \& Perez, 2010).

Therefore, this virus was rapidly adapting with had a potential to further spread into other farms, if it had not been detected on time.

The genetic changes occurred in the viral genome within a short time window 
(less than a month) allowed to correctly group Farm A and B clusters in the phylogeny reconstructed for segments HA, NA PB1 and M. However, the reconstruction of the genetic similarity of the remaining segments did not allow to discern between Farm A and B. This is information is relevant to understand the level of resolution to reconstruct inter farm transmission networks in based on phylogenies.

Because of the rapid diagnostic and intervention, the LPAI outbreak that affected Chilean turkeys in 2017 was contained and limited to only 2 premises of the same company. Phylogenetic analyses revealed that the outbreak was caused by a South American wild bird lineage virus, and unrelated to the 2017 North American H7N9 outbreak in the United States in March. The lack of systematic molecular AIV surveillance in South American countries highlights the need to coordinate regional efforts to better understand the genetic diversity and viral dynamics in the region, and to be prepared for future outbreaks in domestic species (Afanador-Villamizar et al., 2017). This report contributes to understanding this particular clinical LPAI event in turkey and the role of local South American lineages circulating in the wild reservoirs as a major source of strains that are pathogenic to domestic poultry.

\section{ACKNOWLEDGEMENTS}

We thank all personnel from the Virology Unit and Betty Yangari from Biotechnology Unit at the Lo Aguirre Agricultural and Livestock Service Laboratory. B. Brito was funded by the University of Technology Sydney under 
the Chancellor's Research Fellowship Program. V. Neira was funded by Programa

Fondecyt de Iniciación Nº 11170877 and to Programa de Investigación

Asociativa from the Comisión Nacional de Investigación Científica y Tecnológica, project CONICYT-PIA Anillo ACT 1408.

\section{CONFLICT OF INTEREST}

The authors declare that there is no conflict of interest regarding the publication of this article.

\section{REFERENCES}

Afanador-Villamizar, A., Gomez-Romero, C., Diaz, A., \& Ruiz-Saenz, J. (2017). Avian influenza in Latin America: A systematic review of serological and molecular studies from 2000-2015. PLoS One, 12(6), e0179573. doi:10.1371/journal.pone.0179573

Alexander, D. J. (2000). A review of avian influenza in different bird species. Vet Microbiol, 74(1-2), 3-13. doi.org/10.1016/S03781135(00)00160-7

Baele, G., Li, W. L., Drummond, A. J., Suchard, M. A., \& Lemey, P. (2013). Accurate model selection of relaxed molecular clocks in bayesian phylogenetics. Mol Biol Evol, 30(2), 239-243. doi: $10.1093 / \mathrm{molbev} / \mathrm{mss} 243$

Bravo-Vasquez, N., Di Pillo, F., Lazo, A., Jimenez-Bluhm, P., SchultzCherry, S., \& Hamilton-West, C. (2016). Presence of influenza viruses in backyard poultry and swine in El Yali wetland, Chile. Prev Vet Med, 134, 211-215. doi:10.1016/j.prevetmed.2016.10.004

Campitelli, L., Mogavero, E., De Marco, M. A., Delogu, M., Puzelli, S., Frezza, F., . . . Donatelli, I. (2004). Interspecies transmission of an H7N3 influenza virus from wild birds to intensively reared domestic poultry in Italy. Virology, 323(1), 24-36.

doi:10.1016/j.virol.2004.02.015

CDC, Centers for Disease Control and Prevention. (2018). Asian Lineage Avian Influenza A (H7N9) Virus. Retrieved from https://www.cdc.gov/flu/avianflu/h7n9-virus.htm

Djunaidi, H., \& Djunaidi, A. (2007). The Economic Impacts of Avian Influenza on World Poultry Trade and the U.S. Poultry Industry: A 
Spatial Equilibrium Analysis. Journal of Agricultural and Applied Economics, 39(2), 313-323. doi:10.1017/S1074070800023014

Drummond, A. J., Suchard, M. A., Xie, D., \& Rambaut, A. (2012). Bayesian phylogenetics with BEAUti and the BEAST 1.7. Mol Biol Evol, 29(8), 1969-1973. doi:10.1093/molbev/mss075

Edgar, R. C. (2004). MUSCLE: a multiple sequence alignment method with reduced time and space complexity. BMC Bioinformatics, 5, 113. doi: $10.1186 / 1471-2105-5-113$

Jiao, P., Song, Y., Huang, J., Xiang, C., Cui, J., Wu, S., . . . Liao, M. (2018). H7N9 Avian Influenza Virus Is Efficiently Transmissible and Induces an Antibody Response in Chickens. Front Immunol, 9, 789. doi: 10.3389/fimmu.2018.00789

Jimenez-Bluhm, P., Karlsson, E. A., Freiden, P., Sharp, B., Di Pillo, F., Osorio, J. E., . . . Schultz-Cherry, S. (2018). Wild birds in Chile Harbor diverse avian influenza A viruses. Emerg Microbes Infect, 7(1), 44. doi:10.1038/s41426-018-0046-9

Ke, C., Mok, C. K. P., Zhu, W., Zhou, H., He, J., Guan, W., . . . Peiris, J. S. M. (2017). Human Infection with Highly Pathogenic Avian Influenza A(H7N9) Virus, China. Emerg Infect Dis, 23(8), 13321340. doi: $10.3201 /$ eid2308.170600

Kumar, S., Stecher, G., \& Tamura, K. (2016). MEGA7: Molecular Evolutionary Genetics Analysis Version 7.0 for Bigger Datasets. Mol Biol Evol, 33(7), 1870-1874. doi:10.1093/molbev/msw054

Lee, D. H., Torchetti, M. K., Killian, M. L., Berhane, Y., \& Swayne, D. E. (2017). Highly Pathogenic Avian Influenza A(H7N9) Virus, Tennessee, USA, March 2017. Emerg Infect Dis, 23(11). doi: $10.3201 /$ eid2311.171013

Li, J., Zu Dohna, H., Cardona, C. J., Miller, J., \& Carpenter, T. E. (2011). Emergence and genetic variation of neuraminidase stalk deletions in avian influenza viruses. PLoS One, 6(2), e14722.

doi:10.1371/journal.pone.0014722

Mathieu, C., Moreno, V., Pedersen, J., Jeria, J., Agredo, M., Gutierrez, C., . . . Retamal, P. (2015). Avian Influenza in wild birds from Chile, 2007-2009. Virus Res, 199, 42-45. doi:10.1016/j.virusres.2015.01.008

Mathieu, C., Moreno, V., Retamal, P., Gonzalez, A., Rivera, A., Fuller, J., . . . Avalos, P. (2010). Pandemic (H1N1) 2009 in breeding turkeys, Valparaiso, Chile. Emerg Infect Dis, 16(4), 709-711. doi:10.3201/eid1604.091402

Max, V., Herrera, J., Moreira, R., \& Rojas, H. (2007). Avian influenza in Chile: a successful experience. Avian Dis, 51(1 Suppl), 363-365. doi:10.1637/7631-042806R1.1

Miller, M. A., Pfeiffer, W., \& Schwartz, T. (2010, 14, Nov, 2010). Creating the CIPRES Science Gateway for inference of large phylogenetic trees. Paper presented at the Proceedings of the Gateway Computing Environments Workshop (GCE), New Orleans. 
OIE, World Organization for Animal Health (2017). Wahis, Follow-up report No. 8 (Final report). World Animal Health Information Database Retrieved from

https://www.oie.int/wahis_2/public/wahid.php/Reviewreport/Revie w ?reportid $=24038$

OIE, World Organization for Animal Health (2018a). OIE Situation Report for Avian Influenza. Retrieved from http://www.oie.int/fileadmin/Home/eng/Animal_Health_in_the_Wor Id/docs/pdf/OIE_AI_situation_report/OIE_SituationReport_AI_Janua ry2018_01.pdf

OIE, World Organization for Animal Health (2018b). Update on avian influenza in animals (types $\mathrm{H} 5$ and H7), Avian Influenza Portal. Retrieved from http://www.oie.int/animal-health-in-theworld/update-on-avian-influenza/

Otte, J., Hinrichs, J., Rushton, J., D, R.-H., \& D, Z. (2008). Impacts of avian influenza virus on animal production in developing countries. CAB Reviews: Perspectives in Agriculture, Veterinary Science, Nutrition and Natural Resources, 3(80). doi: 10.1079/PAVSNNR20083080

SAG, Servicio Agricola y Ganadero. (2011). Informe de Situacion Sanitaria de Chile Año 2011. Retrieved from https://www2.sag.gob.cl/Pecuaria/bvo/BVO 15 I semestre 2012/a uehacer unidades/descargables/situacion sanitaria animal 2011.p df. Last accessed December 21, 2018.

Sorrell, E. M., Song, H., Pena, L., \& Perez, D. R. (2010). A 27-amino-acid deletion in the neuraminidase stalk supports replication of an avian $\mathrm{H} 2 \mathrm{~N} 2$ influenza A virus in the respiratory tract of chickens. J Virol, 84(22), 11831-11840. doi:10.1128/JVI.01460-10

Spackman, E., Gelb, J., Jr., Preskenis, L. A., Ladman, B. S., Pope, C. R., Pantin-Jackwood, M. J., \& McKinley, E. T. (2010). The pathogenesis of low pathogenicity $\mathrm{H} 7$ avian influenza viruses in chickens, ducks and turkeys. Virol J, 7, 331. doi:10.1186/1743-422X-7-331

Spackman, E., McCracken, K. G., Winker, K., \& Swayne, D. E. (2006). H7N3 avian influenza virus found in a South American wild duck is related to the Chilean 2002 poultry outbreak, contains genes from equine and North American wild bird lineages, and is adapted to domestic turkeys. J Virol, 80(15), 7760-7764.

doi:10.1128/JVI.00445-06

USDA, United States Department of Agriculture, Veterinary Services National Preparedness and Incident Coordination NPIC. (2015). Highly Pathogenic Avian Influenza (HPAI). Incidence 2014-2015, Ready Reference Guide- Overview of Zones. Retrieved from https://www.aphis.usda.gov/animal health/emergency manageme nt/downloads/hpai zones.pdf. Last accessed December 21, 2018

Vandegrift, K. J., Sokolow, S. H., Daszak, P., \& Kilpatrick, A. M. (2010). Ecology of avian influenza viruses in a changing world. Ann $N Y$ Acad Sci, 1195, 113-128. doi:10.1111/j.1749-6632.2010.05451.x 


\begin{tabular}{|c|c|c|c|c|c|}
\hline zo & $\stackrel{T}{\triangleright}$ & $D$ & 邑 & $\underset{\sim}{\stackrel{D}{N}}$ & 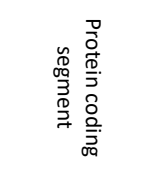 \\
\hline 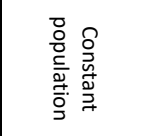 & 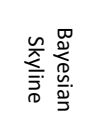 & 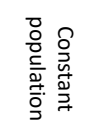 & 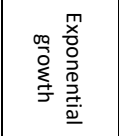 & 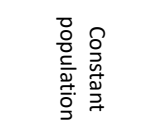 & 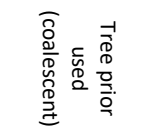 \\
\hline 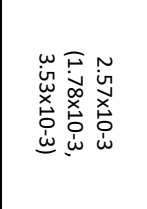 & 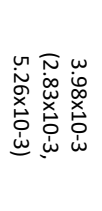 & 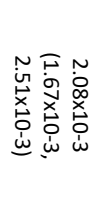 & 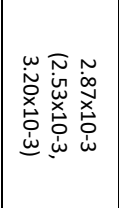 & 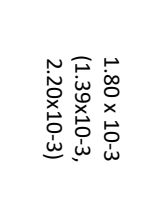 & 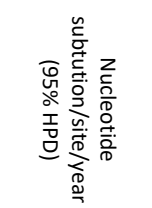 \\
\hline 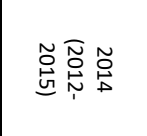 & 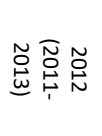 & 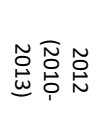 & 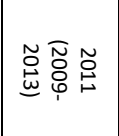 & 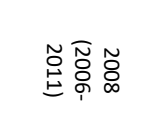 & 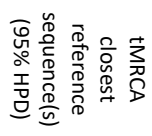 \\
\hline 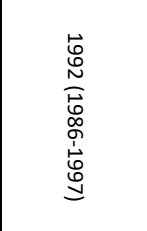 & $\begin{array}{l}\vec{b} \\
\stackrel{0}{0} \\
u \\
\overrightarrow{0} \\
o \\
0 \\
\dot{0} \\
\stackrel{0}{0}\end{array}$ & $\begin{array}{l}\vec{b} \\
\stackrel{0}{0} \\
0 \\
\overrightarrow{0} \\
0 \\
0 \\
\dot{0} \\
0 \\
0 \\
0\end{array}$ & 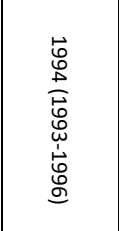 & 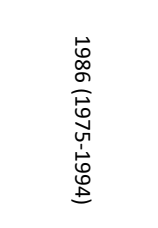 & 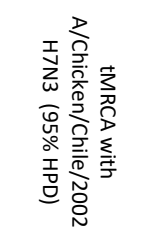 \\
\hline 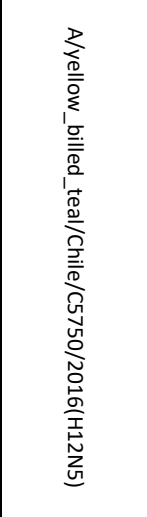 & 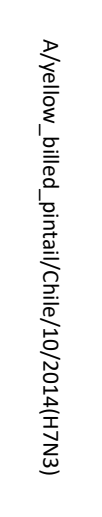 & 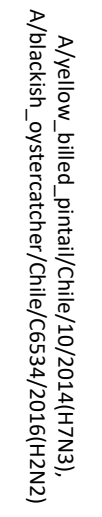 & 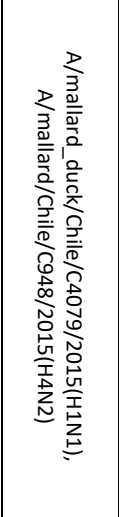 & 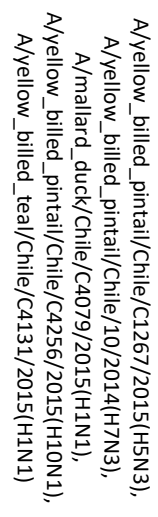 & 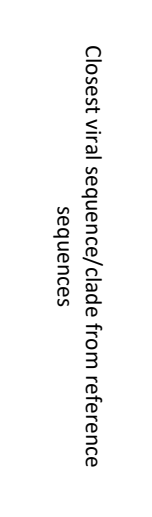 \\
\hline 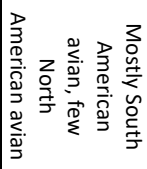 & 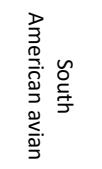 & 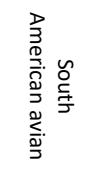 & 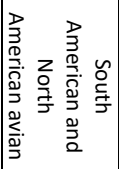 & 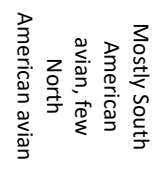 & 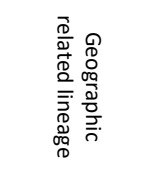 \\
\hline 点 & * & 点 & * & * & 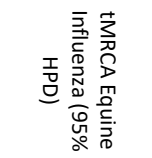 \\
\hline
\end{tabular}

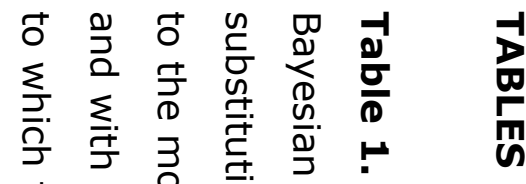

守

吕

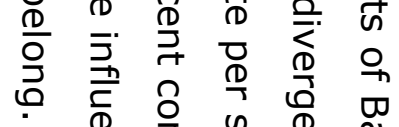

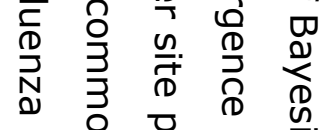

؛ 익

空

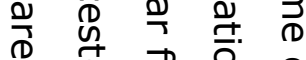

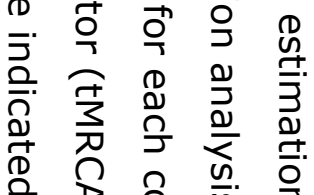

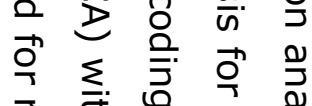

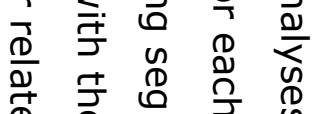

莡 宁 号

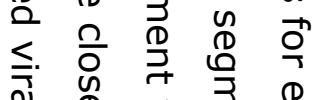

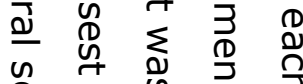

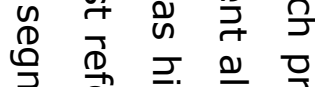

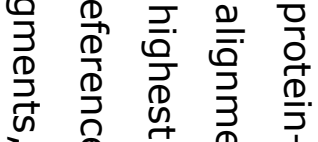

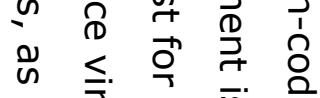

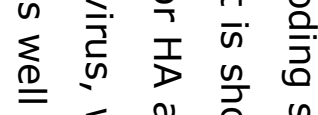

离 站

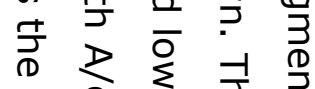

곰 술 仓

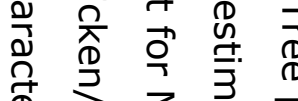

疍.

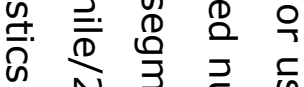

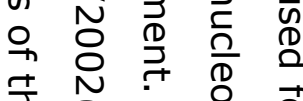

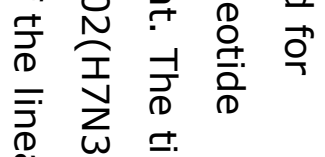




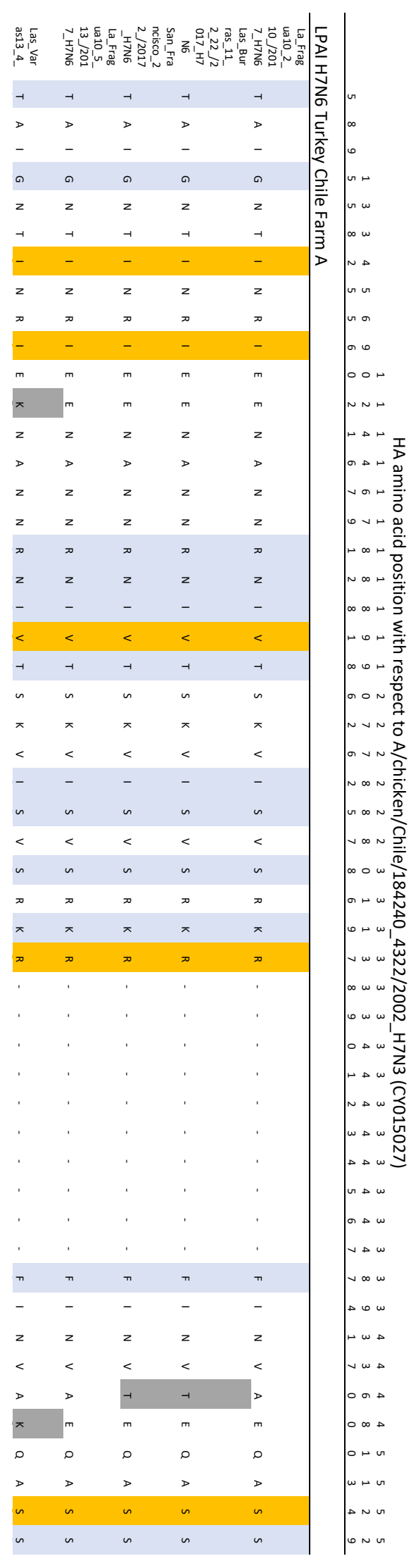

$$
\checkmark \vec{\sigma} \vec{u} \vec{\oplus} \vec{\omega}=\overrightarrow{0}
$$

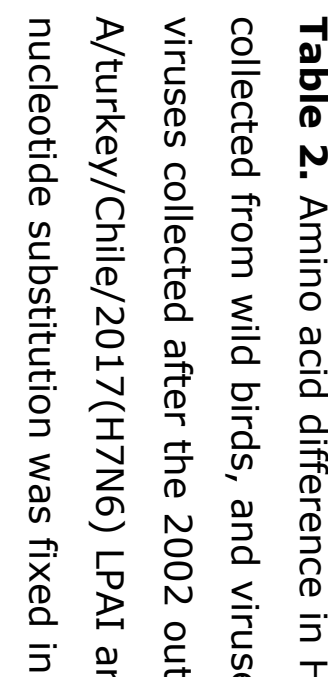

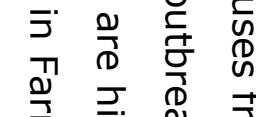

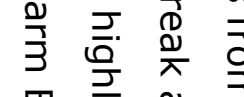

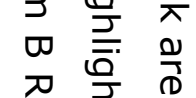$$
\underset{\omega}{\omega} \stackrel{\vec{D}}{\sigma}
$$$$
\text { 홋 }
$$

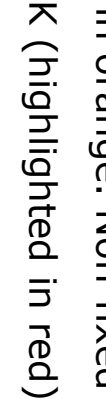$$
\text { 응 }
$$$$
\text { 응 }
$$$$
\text { 万人 }
$$

ถ

วิ่

ค อ

ज 节完

$\stackrel{\varphi}{\stackrel{D}{\gtrless}}$

ㅇำ

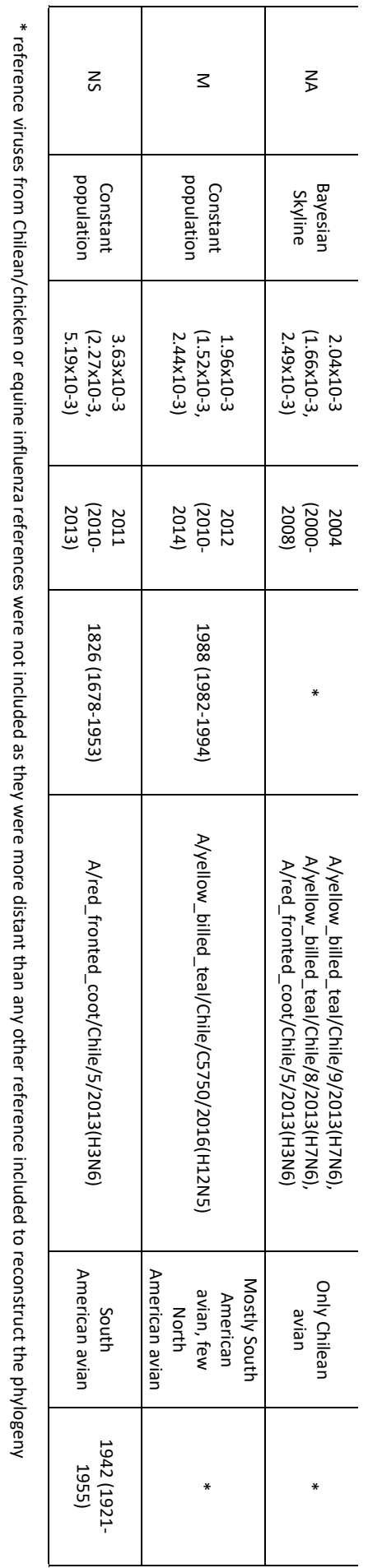


bioRxiv preprint doi: https://doi.org/10.1101/527069; this version posted January 26,2019 . The copyright holder for this preprint (which was not certified by peer review) is the author/funder. All rights reserved. No reuse allowed without permission.

$\sim N \sigma \infty$

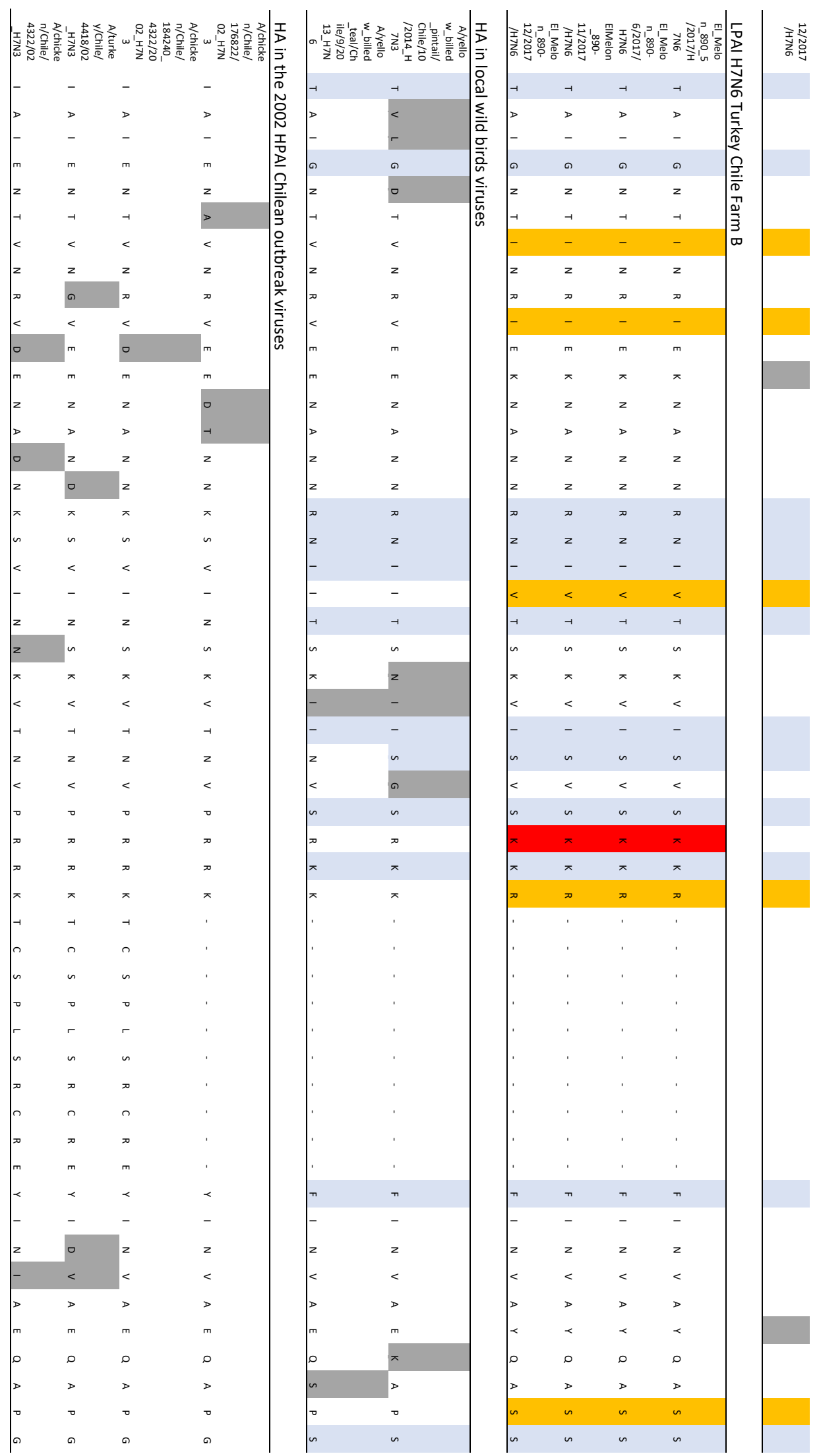




\section{FIGURE LEGENDS}

24 Figure 1. Maximum clade credibility tree of HA segment. The South American

25 lineage shared a common ancestor in 1944 (95\% HPD 1913-1969) with the closest

26 reference viruses (North American Lineage). The viruses that caused the H7N6 LPAI

27 outbreak in 2017 in Chile were genetically related to the viruses that caused an

28 HPAI outbreak in 2002 in Chile (A/chicken/Chile/2002(H7N3)).

30 Figure 2. Maximum clade credibility tree of NA segment.

$31 \mathrm{~A} /$ turkey/Chile/2017(H7N6) LPAI viruses are grouped in a unique cluster unrelated

32 to other references and sharing a common ancestor in 1869 (95\% HPD 1823-1911)

33 with the closest North American lineage.

35 Figure 3. Maximum clade credibility tree of segments PB2, PB1, PA, NP, M and NS.

36 All segments are closely related to viruses previously collected from Chilean wild

37 birds. All Segments, except for NS, are related to the previous Chilean 2002 HPAI

38 outbreak.

40 Supplementary file 1. Antigenic sites of in HA sequences of reference viruses and 41 viruses collected from poultry and wild birds in Chile. 

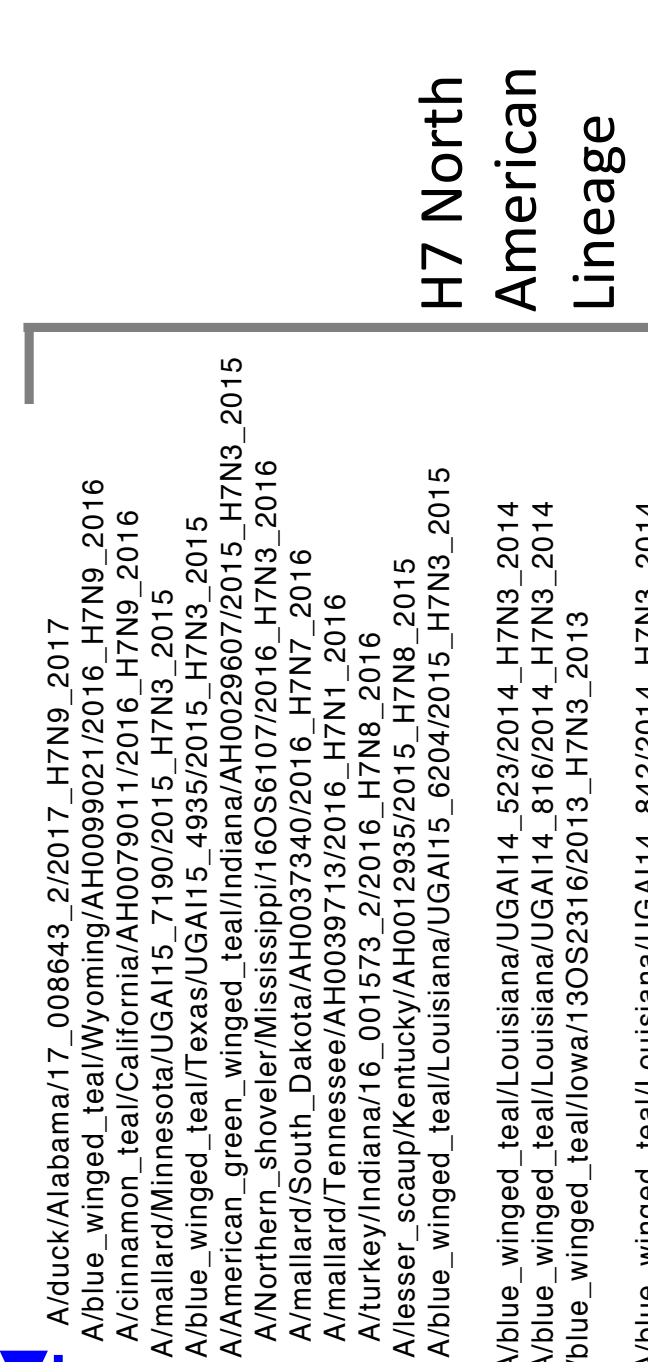

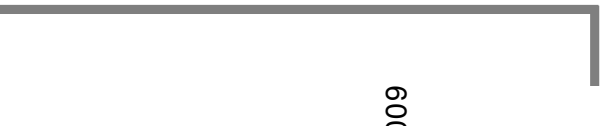

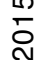

¿

$\sum^{\prime} \sum^{\prime} m \quad \sum^{\prime} \infty \mathscr{O}^{\prime}$

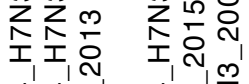

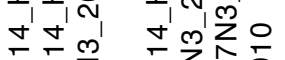

온초

के

ஸिक m

$\nabla_{1}+\frac{1}{2}$

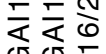

णำ

๙

๘

क⿻ 丨⿴囗十

옹

ब。 ब

$\pm \Phi$

o 8

育.

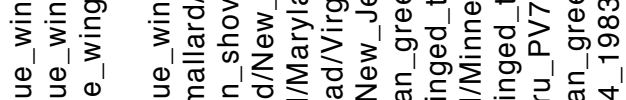

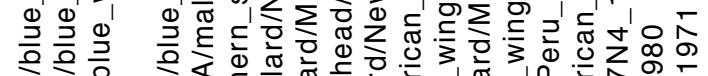

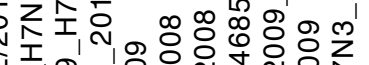

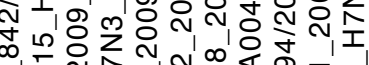

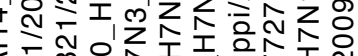

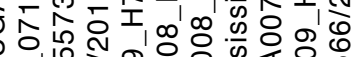

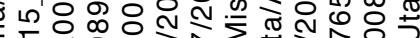

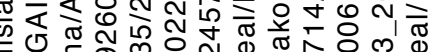
○ว.匹 ब

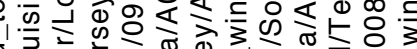

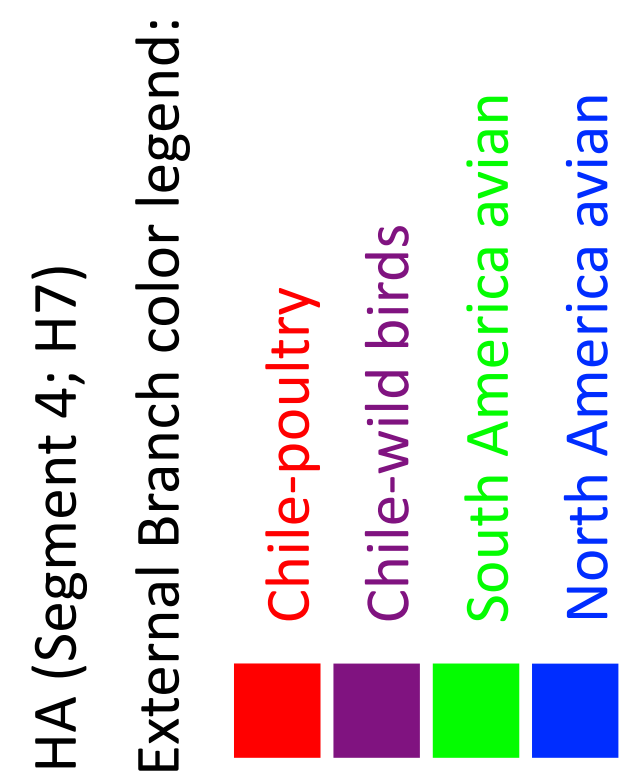

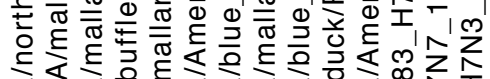

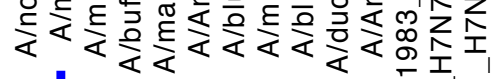

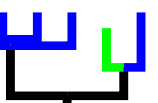

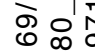

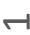

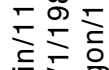

$\infty$ $\stackrel{0}{z} \frac{0}{\frac{1}{v}}$

I $>$

๘

๙ $\frac{\varepsilon}{\bar{\delta}}$

(1) 
bioRxiv preprint doi: https://doi.org/10.1101/527069; this version posted January 26, 2019. The copyright holder for this preprint (which was not certified by peer review) is the author/funder. All rights reserved. No reuse allowed without permission.

NA (Segment 6; N6)

External Branch color legend:

Chile-poultry

Chile-wild birds

South America avian

North America avian

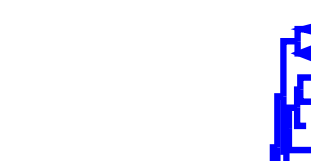

A/mallard/California/1188/2010 H4N6 2010

- A/mallard/Alaska/849/2013_H4N6_2013

A/mallard/California/5271/2009 H4N6 2009

A/blue winged teal/Texas/Al11 3139/2011 mixed 2011

L A/mallard/California/7766/2008_H4N6_2008

C A/mallard/Interior_Alaska/4/2007_H4N6_2007

A/blue winged_AlT/Al12 3768/2012 H11N6 2012

- A/redhead/Ohio/13OS0373/2013_H3N6_2013

I. A/blue_winged_teal/Guatemala/CIP049H106_62/2011_H14N6_2011

A/mallard/Maryland/08OS3079/2008 H4N6_2008

A/environment/Maryland/1101/2006 H4N6 2006

A/mallard/Maryland/060S1110/2006 H4N6 2006

- A/mallard/Interior Alaska/5/2007 H4 N6 2007

C A/northern pintail/Interior_Alaska/1/2007_H4N6_2007

LA/pintail/Alberta/166/2003 H3N6 2003

L A/mallard/Alberta/125/1999_H11N6 1999

A/pintail/Alberta/269/2001_H4N6_2001

A/pintail/Alberta/269/2001_H4N6_2001

[_A/gull/Peru/CH134/2010_HN6_2010

A/mallard/Alberta/237/2003_H4N6_2003

Influenza_A virus_A/mallard/Alberta/295/98 H4N6 1998

- A/mallard/Minnesota/Sg_00133/2007_H4N6_2007

$L$ A/blue winged teal/Alberta/293/2003 H4N6 2003

A/northern_shoveler/California/27985/2007_H7N6_2007

A/emperor_goose/Alaska/44045_125/2006_H4N6_2006

C A/mallard/MN/99/2000_H3N6_2000

Influenza_A virus A/mallard/Alberta/199/1999_H3N6_1999

A/mallard/Alberta/199/1999 H3N6 1999

A/pintail/Alberta/275/1996 H4N6 1996

A/mallard/ALB/49/1995 H4N6 1995

-

A/mallard/Alberta/144/1987_mixed_1987
A/redhead_duck/ALB/116/1985_H4N6_1985
A/pintail_duck/ALB/462/1979_H3N6_1979

A/pintail_duck/ALB/462/1979_H3N6_1979

A/pintail_duck/ALB/159/1977_H4N6_1977

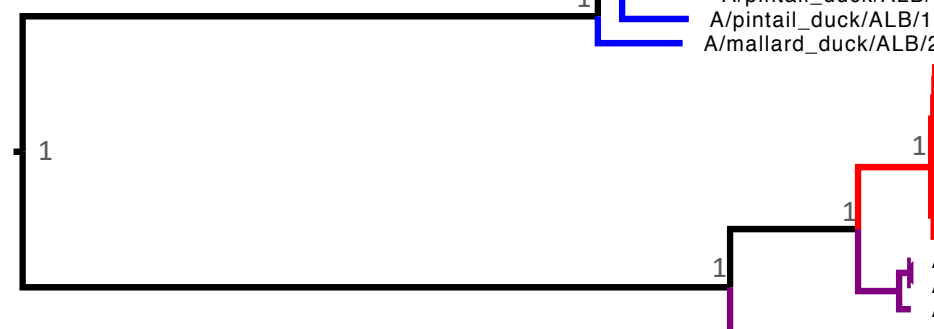

A/turkey/Chile/La_Fragua_10_2_10_/2017_H7N6_NA_2017 A/turkey/Chile/La_Fragua $10 \quad 5 \quad 13$ /2017 H7N6 NA 2017

A/turkey/Chile/Las Burras 11_2 22/2017 H7N6 NA 2017 A/turkey/Chile/San_Francisco_22/2017 H7N6_NA_2017

A/turkey/Chile/EI Melon 890-12/H7N6 2017

A/turkey/Chile/EI_Melon_890-6/H7N6_2017

A/turkey/Chile/EI_Melon_890-11/H7N6_2017

A/turkey/Chile/EI Melon 890 5/H7N6 2017

A/yellow_billed teal/Chile/9/2013_H7N6 2013

A/yellow billed teal/Chile/8/2013 H7N6 2013

A/red fronted coot/Chile/5/2013_H3N6 2013

A/yellow billed pintail/Chile/7/2013_H4N6 2013

A/yellow_billed_pintail/Chile/6/2013_H4N6_2013

H7 North

American

Lineage
FarmA LPAI H7N6

Turkey

FarmB Chile 2017

) $1880 \quad 1900 \quad 1920 \quad 1940 \quad 1960 \quad 1980 \quad 2000 \quad 2020$ 
bioRxiv preprint doi: https://doi.org/10.1101/527069; this version posted January 26, 2019. The copyright holder for this preprint (which was not certified by peer review) is the author/funder. All rights reserved. No reuse allowed without permission.

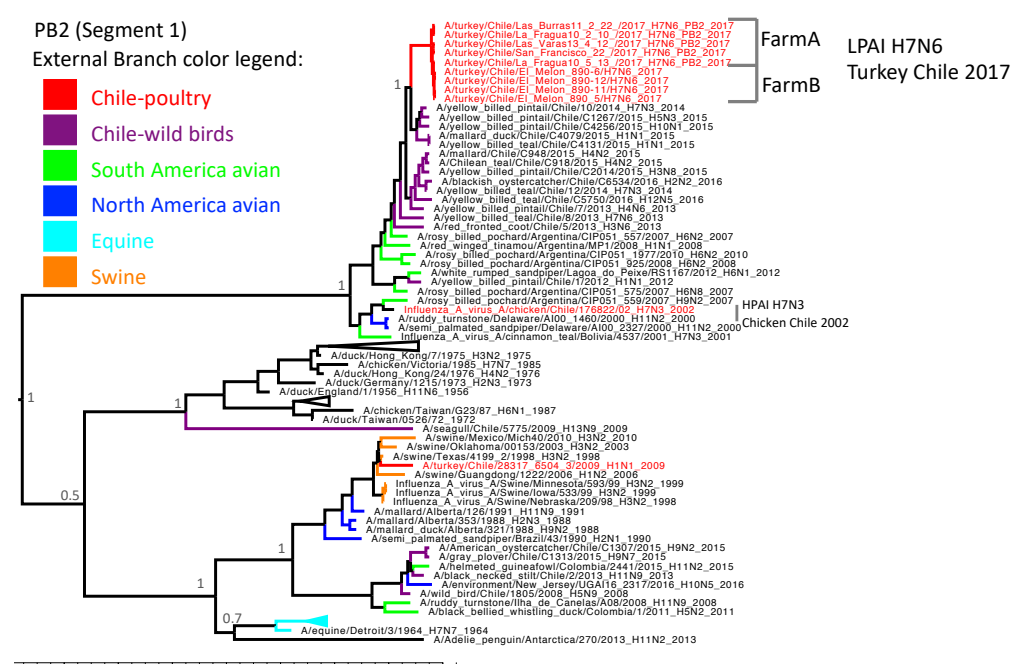

$\begin{array}{lllllllll}30 & 1880 & 1900 & 1920 & 1940 & 1960 & 1980 & 2000 & 2020\end{array}$

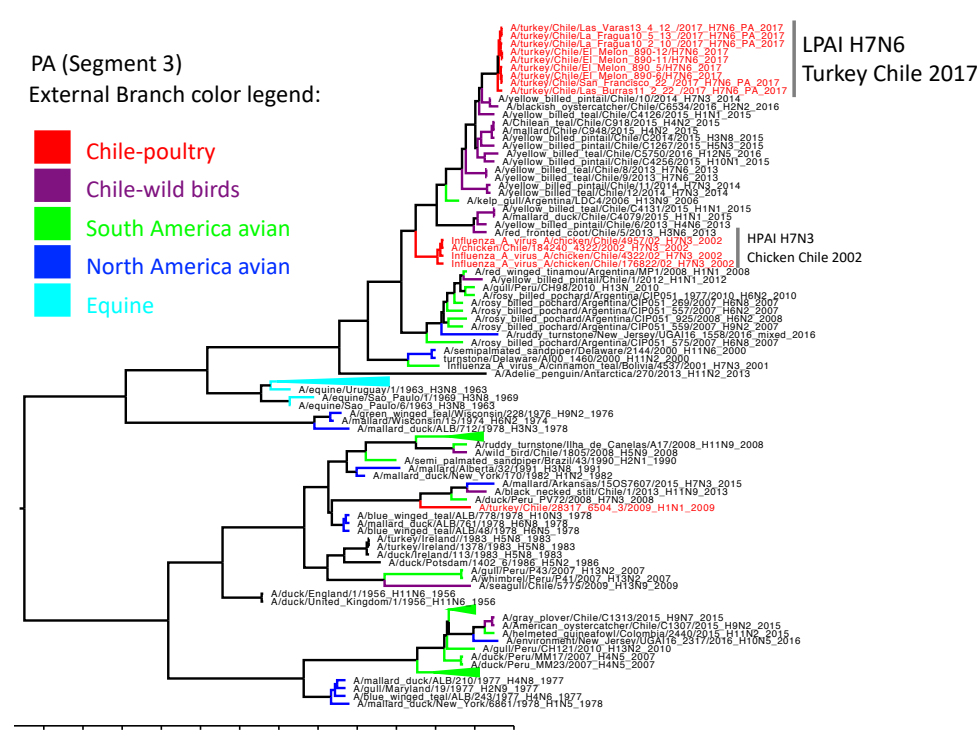

$\widetilde{01900191019201930194019501960197019801990200020102020 \text { : }}$

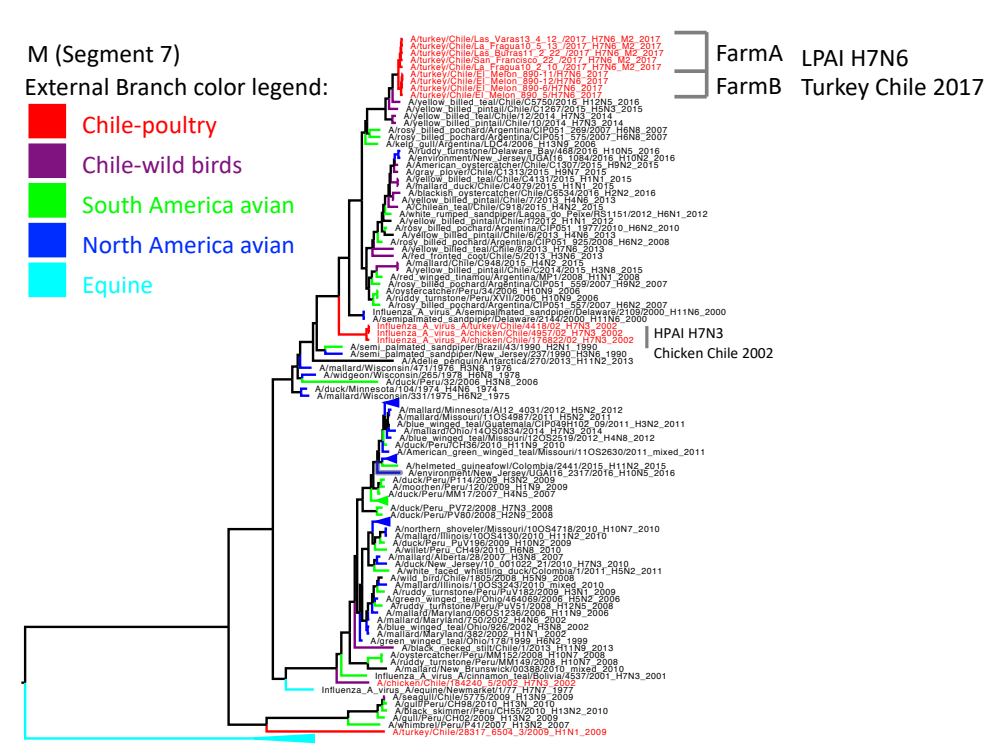

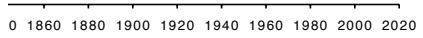

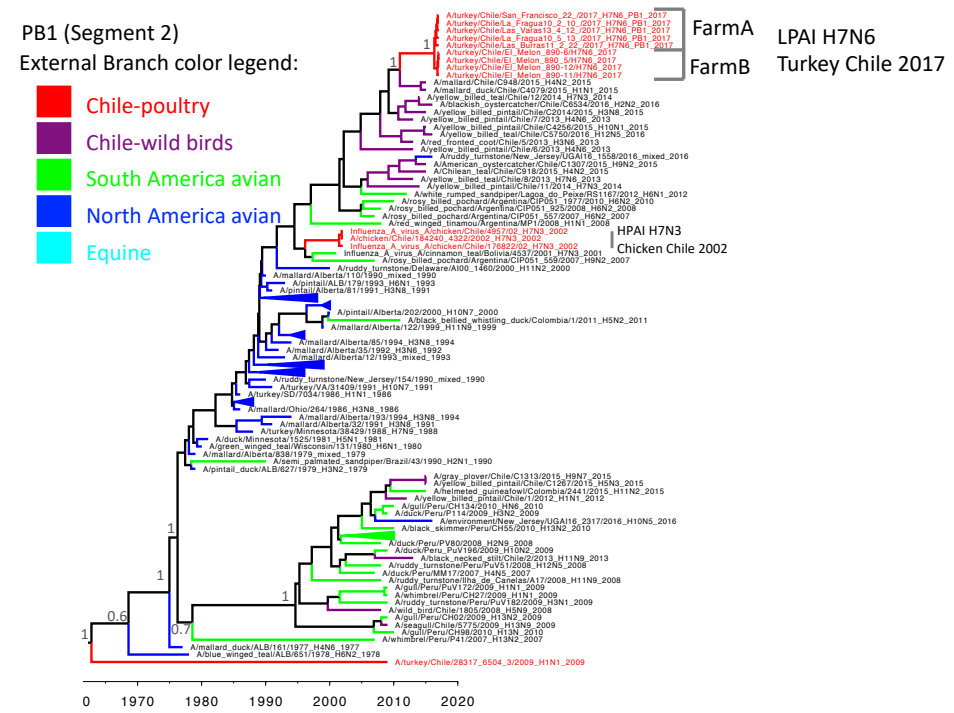

NP (Segment 5)

External Branch color legend:

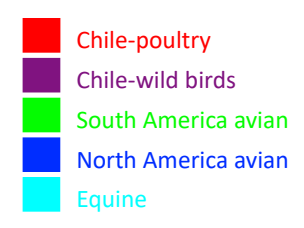

LPAI H7N6 Turkey Chile 2017

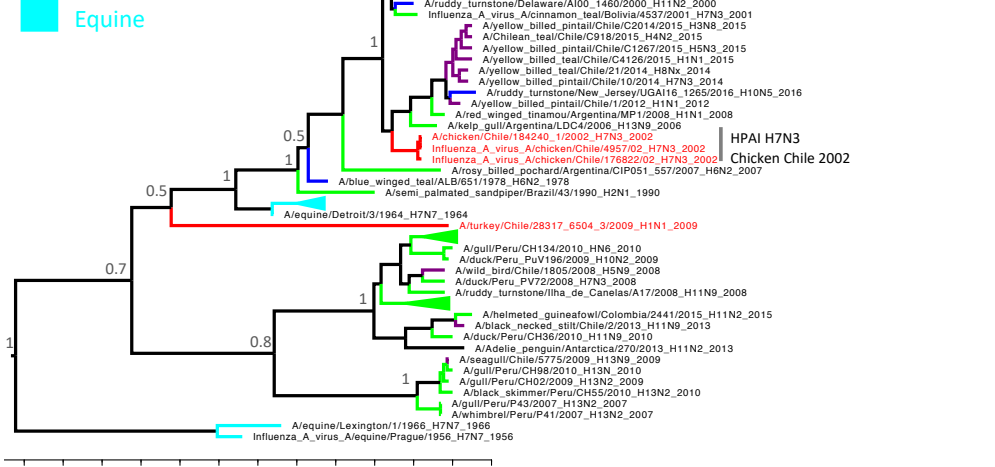

$190019101920193019401950196019701980199020002010202 C$

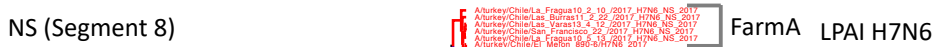
External Branch color legend: $\quad \begin{array}{lll}1 \\ \text { and }\end{array}$

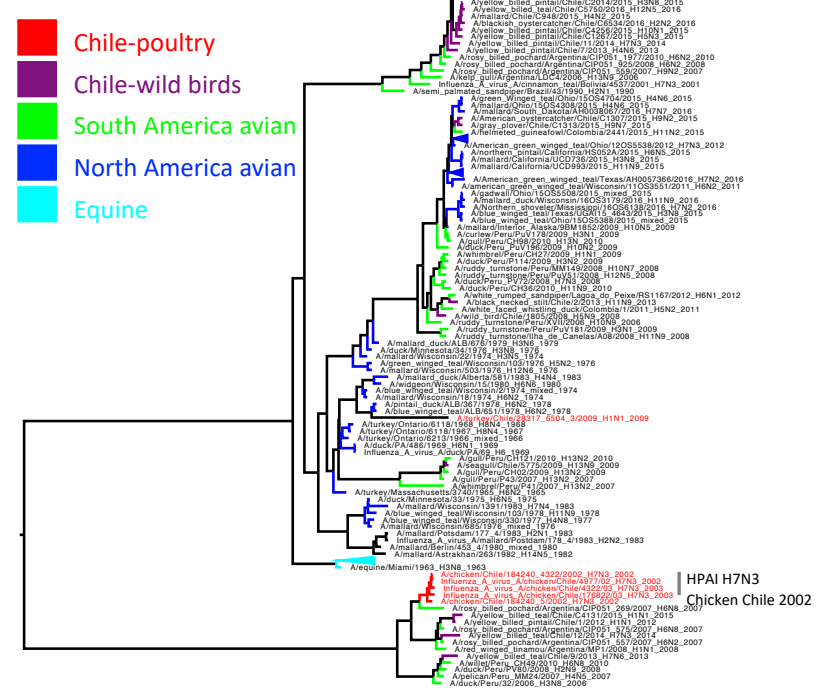

10 $\begin{array}{llllllllll}1840 & 1860 & 1880 & 1900 & 1920 & 1940 & 1960 & 1980 & 2000 & 2020\end{array}$ 